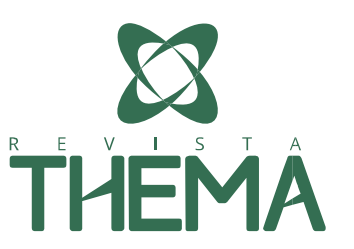

\title{
Integração Entre as Disciplinas de Desenho Técnico I e Informática Básica Auxiliado por Software Gráfico em Curso Técnico
}

\section{Integration Between the subjects of Technical Drawing I and Basic Informatics Aided by Graphic Software in a technical course}

Luiz Henrique Neves Pachecoㅁ; Janete Otte ${ }^{1}$

\section{RESUMO}

Neste artigo apresenta-se o resultado do trabalho que está sendo realizado no Curso Técnico de Mecânica do Instituto Federal Sul-rio-grandense, câmpus Pelotas, nas disciplinas de Desenho Técnico I e Informática Básica, em que se utiliza o software Solidworks ${ }^{\circledR}$ com o objetivo de melhorar a aprendizagem do estudante quanto à visualização espacial de um sólido, por conseguinte, o aprendizado de desenho técnico. São apresentados alguns referenciais teóricos sobre o desenho técnico e o software específico para desenvolvê-lo através do uso de computadores no auxílio do aprendizado. A partir de uma pesquisa com os professores que ministram as disciplinas de Informática Básica e de Desenho Técnico I, relata-se as suas opiniões e percepções sobre a superação das dificuldades de aprendizado dos estudantes na área de desenho e suas sugestões de aperfeiçoamento do método utilizado. Conclui-se que é necessária maior integração entre as disciplinas para possibilitar aos alunos uma melhor compreensão da visualização espacial e, por conseguinte, da prática de desenho técnico.

Palavras-chave: Integração; Desenho Técnico I; Informática Básica; Visualização espacial.

\begin{abstract}
This article presents the work being carried out in the Mechanical Engineering Course of the Federal Institute of Sul-rio-grandense of Pelotas Campus, in the subjects of Technical Design I and Basic Informatics. In this study, Solidworks ${ }^{\circledR}$ software is used with the objective to improve students' learning regarding the spatial visualization of a solid, consequently, the learning of technical drawing. Some theoretical references about the technical design and the specific software are presented to develop it through the use of computers to aid in learning. From a research with the teachers that teach the subjects of Basic Informatics and Technical Drawing $I$, it is concluded that greater integration between disciplines is necessary to enable students to better understand spatial visualization and, therefore, the practice of technical drawing. In this research, the teachers' opinions and suggestions regarding this form of joint work were reported, with the objective of overcoming the learning difficulties of the students in the drawing area.
\end{abstract}

Keywords: Integration; Technical Drawing I; Basic Informatics; Spatial visualization.

\footnotetext{
${ }^{1}$ IFSul - Instituto Federal de Educação, Ciência e Tecnologia Sul-rio-grandense, Pelotas/RS - Brasil.
} 


\section{INTRODUÇÃO}

Tem-se observado que na disciplina de Desenho Técnico I, do primeiro módulo do Curso Técnico em Mecânica, câmpus Pelotas do Instituto Federal de Educação, Ciência e Tecnologia Sul-rio-grandense (IFSul), nas modalidades concomitante e subsequente, acontece um alto índice de reprovação. Um dos fatores é a grande dificuldade dos alunos para representar graficamente, em forma bidimensional ou tridimensional, os sólidos ou peças mecânicas apresentadas nos exercícios propostos.

Desta forma passou-se a partir do primeiro semestre de 2015 a realizar um trabalho conjunto entre as disciplinas de Desenho Técnico I e de Informática Básica, em que se utiliza o software Solidworks ${ }^{\circledR}$ com o objetivo de melhorar a aprendizagem do estudante quanto à visualização espacial, considerando as possibilidades que o Solidworks ${ }^{\circledR}$ tem de gerar um sólido ou peça mecânica em perspectiva, facilitando o entendimento do aluno.

O Curso Técnico de Mecânica é um dos nove cursos técnicos oferecidos pelo câmpus Pelotas do Instituto Federal Sul-rio-grandense. Atualmente, segundo o sistema acadêmico da instituição, o número de matrículas no curso Técnico em Mecânica no câmpus Pelotas, é de 280 e, destas, 50\% são do primeiro módulo. O estudante que reprova em até duas disciplinas, pode avançar de semestre e realizar essas disciplinas paralelamente, chamadas de dependência. A disciplina de Desenho Técnico registra um grande número das dependências, resultante da necessidade de os alunos a repetirem.

O conteúdo ministrado nessa disciplina é uma ferramenta importante de comunicação entre os departamentos de uma indústria, exigindo dos profissionais que atuam na área a capacidade de analisar e interpretar um desenho técnico. Com relação à importância do desenho técnico, Sulz e Silva (2013) afirmam que:

O Desenho foi elemento indispensável à implantação, sustentação e expansão da industrialização, e permanece como competência relevante na formação de profissionais envolvidos na produção industrial, especialmente os que ocupam os mais altos níveis hierárquicos da cadeia produtiva mundial. 0 Desenho está intrinsecamente ligado ao crescimento econômico, posto que o crescimento econômico é pautado na ênfase dada à formação de capital humano, pelo desenvolvimento tecnológico, estes reforçados pela acumulação de capital (SULZ e SILVA, 2013, p.10).

Na disciplina de Informática Básica, onde se se utilizam computadores para o ensino, tem-se um fator que facilita para o professor o ensinar e para o aluno o aprender, como indicam Hellmeister, Deganutti e Rossi (2013):

Os alunos de hoje estão habituados a vídeos-game, jogos interativos e em rede, internet, sites de relacionamento, estão sempre plugados, on-line, ao mesmo tempo estão ouvindo música, lendo e-mails, se relacionando via redes sociais e por incrível que pareça, estudando (HELLMEISTER, DEGANUTTI e ROSSI, 2013, p.3).

Dessa forma, na proposta de integração das disciplinas, dentro das especificidades de cada uma, procura-se somar os conhecimentos obtidos com os diferentes recursos utilizados, visto que cada uma trabalha habilidades específicas necessárias ao aprendizado. Sobre isso concorda-se com Marques e Chisté (2016) quando se referem ao ensino do desenho técnico, como segue:

Os autores defendem uma metodologia que une os instrumentos tradicionais de Desenho Técnico à computação gráfica e alertam que a simples substituição do desenho instrumental pelo assistido por computador pode ter como resultado um 
prejuízo na formação da visão espacial e no raciocínio do aluno, devido à falta de manipulação dos instrumentos e falta do traçado manual do desenho (KEMPTER et al 2012 apud MARQUES e CHISTÉ, 2016, p.1180).

E, do mesmo modo, com relação ao uso de computador, quando os mesmos autores citam o seguinte:

Em contrapartida, também salientam como o computador pode enriquecer a aula, fortalecendo o conhecimento da Geometria Descritiva, contribuindo com a elaboração de objetos tridimensionais, proporcionando dinamismo às aulas, necessários na atualidade (KEMPTER et al 2012 apud MARQUES e CHISTÉ, 2016, p.1180).

No primeiro módulo na disciplina de Desenho Técnico I, verifica-se a dificuldade do aluno em conseguir ter uma visão espacial de um sólido ou de uma peça mecânica e representá-los em diferentes formas, tais como em perspectiva isométrica, nas vistas do sistema universal de projeções, com seus detalhes externos e seus detalhes internos. Vários autores citam a importância de os alunos terem a capacidade de visualização espacial desenvolvida. Com relação à dificuldade desse tipo de visualização dos alunos, Valente e Pereira (2015) indicam que:

A Aptidão Espacial necessária na engenharia, na matemática e em outras áreas do conhecimento. Embora essencial na formação do profissional, há uma carência de desenvolvimento desta habilidade cognitiva por parte do discente egresso do Ensino Médio. Isso se apresenta como um desafio para os docentes, principalmente aqueles que lidam com cursos das áreas gráficas, mas buscam superar tal obstáculo, propondo aprimoramentos no processo de ensino (VALENTE e PEREIRA, 2015, p.142).

Com relação à possibilidade do desenvolvimento da visualização espacial dos alunos, Montenegro (2005), citado por Marques (2017), confirma que:

A habilidade espacial é uma capacidade humana que pode ser estimulada ou abandonada; no último caso algumas regiões cerebrais passam a se deteriorar ou a exercer outras funções. Porém, quando estimulada, por meio de aplicação direta numa atividade ou por meio de exercícios que evolvam rotação mental de figuras, reconhecimento de rostos, leitura de mapas, analogias de formas, vistas de perspectivas de vários ângulos, interpretação múltipla de uma mesma figura, ocorre o desenvolvimento da visão espacial (MONTENEGRO, 2005 apud MARQUES, 2017, p.4).

Pode-se afirmar que a dificuldade apresentada pelos alunos com relação à visualização espacial tem sido consequência, segundo vários autores, como por exemplo Tavares (2017), de alterações dos currículos educacionais ocorridas com relação à disciplina de Geometria Descritiva, que teve o seu ensino desvalorizado, chegando até a deixar de ser ministrada em muitas escolas. Dessa forma percebe-se que as mudanças sofridas nos currículos referentes ao ensino, relacionadas às percepções de desenho geométrico e visualização espacial, retiraram conteúdos importantes para o aprendizado do desenho técnico.

Tavares (2017), com relação ao ensino do Desenho Geométrico, salienta que:

O ensino do Desenho Geométrico no currículo da educação básica brasileira foi decretado pela de Lei no 20.158 de 30 de junho de 1931, porém sua não obrigatoriedade foi determinada pela Lei no 5.692, das Leis de Diretrizes e Bases Nacionais de 1971, tornando o seu ensino optativo. A partir desta alarmante questão, instituições vêm deixando o ensino da disciplina em segundo plano. Atualmente a grande realidade é a sua extinção da grade escolar de instituições públicas e particulares (TAVARES, 2017, p.1). 
Percebe-se que outros colegas, professores de diferentes cursos técnicos do mesmo câmpus, têm realizado pesquisas a respeito do assunto junto a estudantes e professores que trabalham com esses conteúdos nos seus respectivos cursos como se pode ver no artigo "Tecnologia digital como recurso didático para potencializar o processo de aprendizagem em desenho técnico na educação profissional" de Custódio, Brod e Lopes (2016), escrito a partir de dados do Curso Técnico em Eletromecânica e no projeto de pesquisa "Geometria $x$ Autocad $^{\circledR}$ : Um estudo de caso no curso de Edificações no IFSul - câmpus Pelotas - RS", de Letícia Arruda (2018), que teve como objetivo conhecer as principais dificuldades dos alunos do Curso Técnico em Edificações, na disciplina de Informática Aplicada II.

Na busca por conhecer melhor o meio em que ocorre o desenvolvimento das disciplinas de Desenho Técnico I e Informática Básica no Curso Técnico de Mecânica e de como elas estão interligadas em seus conteúdos realiza-se uma observação participante, com o propósito de aprimorar esta proposta de trabalho conjunto no planejamento e desenvolvimento das duas disciplinas.

Este trabalho apresenta informações de como estão estruturados e são trabalhados os conteúdos das disciplinas de Desenho Técnico I e Informática Básica. No momento seguinte relata dados sobre a metodologia da pesquisa com os professores e o resultado de suas percepções e, na conclusão, são tecidas algumas considerações da pesquisa realizada.

\section{DISCIPLINAS DE DESENHO TÉCNICO I E INFORMÁTICA BÁSICA}

No perfil ${ }^{2}$ do técnico em Mecânica, a construção e interpretação de desenhos técnicos são duas competências importantes para a atuação profissional, bem como estar apto a visualizar os detalhes de uma peça, ter a capacidade de executar um croqui e, posteriormente, transmitir esses detalhes para um desenho técnico da referida peça, para que outros profissionais da empresa consigam interpretar e utilizar as informações recebidas na execução das suas tarefas.

Para o desenvolvimento dessas competências com relação ao desenho técnico, os alunos do curso Técnico em Mecânica têm, no primeiro e no segundo módulos, respectivamente, a disciplina de Desenho Técnico I e Desenho Técnico II.

A dificuldade do aluno em conseguir ter a visão espacial de um sólido e representá-lo em diferentes vistas, com seus detalhes externos visíveis e seus detalhes internos ocultos, apresenta-se logo no início da disciplina de Desenho Técnico I quando são propostas atividades que exigem esta habilidade.

\subsection{Desenho Técnico I}

Para conscientizar os alunos da importância da disciplina de Desenho Técnico I, salienta-se as competências gerais dos técnicos, constantes no item 4.1 do Projeto Pedagógico do curso Técnico de Nível Médio em Mecânica:

\footnotetext{
2 Perfil: Habilitar profissionais técnicos de nível médio em Mecânica, tornando-os capazes de desenvolver e executar atividades relacionadas às áreas de projetos, fabricação e manutenção mecânica. O técnico de nível médio em Mecânica é o profissional legalmente habilitado, capaz de inserir-se em uma empresa ou atividade autônoma, elaborando, detalhando ou executando projetos de construção mecânica e de automação. Domina amplamente conteúdos relacionados à fabricação mecânica como usinagem e programação de máquinas CNC, controle da qualidade, métodos e processos bem como planejamento e execução de planos e procedimentos de manutenção mecânica. Disponível em: <http://pelotas.ifsul.edu.br/ensino/cursos-tecnicos/mecanica>. Acessado em 17/3/2018.
} 
O Técnico de Mecânica, Área Indústria, de acordo com a resolução CNE/CEB 04/99, deverá ter desenvolvido as seguintes competências profissionais gerais: projetar produtos, ferramentas, máquinas e equipamentos, utilizando técnicas de desenho e de representação gráfica com seus fundamentos matemáticos e geométricos (IFSUL, 2008, p.26).

Para trabalhar essas habilidades, a disciplina de Desenho Técnico I tem a sua ementa especificada da seguinte forma:

Leitura e interpretação de desenhos técnicos mecânicos, execução de esboços a mão livre, desenho preliminar e desenho definitivo com o auxílio de instrumentos para desenho técnico (desenhos de conjuntos mecânicos e detalhamento) (IFSUL, 2008, p.34).

Nas três semanas iniciais do primeiro semestre do curso desenvolve-se o conteúdo que parte da argumentação da importância do desenho técnico na construção de peças até chegar à representação gráfica de um sólido no sistema universal de projeções.

No conteúdo sobre o sistema universal de projeções, ministrado na terceira semana do curso, é apresentada a maneira como são geradas as vistas de um sólido no primeiro diedro, que é o padrão adotado pela Associação Brasileira de Normas Técnicas (ABNT).

Para obtenção das vistas considera-se um sólido no centro de uma caixa, cujas faces são os planos de projeção para as imagens obtidas por um observador. As vistas são obtidas como resultado da projeção da visão de um observador, depois o objeto e na parte posterior o plano para a projeção da imagem, ver Figura 1.

Figura 1 - Representação das vistas de um sólido.

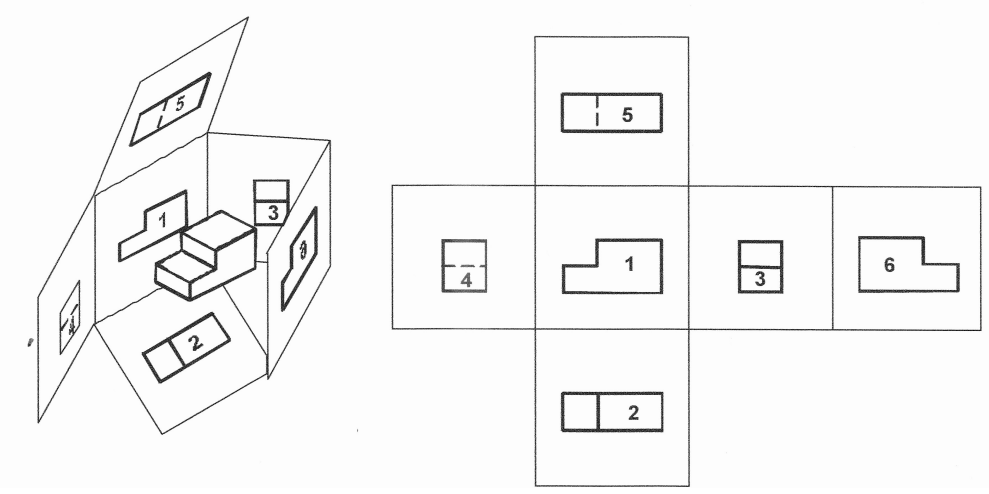

Fonte: Sistemas de projeções ortogonais. (2018)

No primeiro diedro tem-se os seguintes planos: plano 1 - vista frontal ou elevação - mostra a projeção de frente do sólido; plano 2 - vista superior ou planta - mostra a projeção do sólido visto por cima; plano 3 - vista lateral esquerda ou perfil - mostra o sólido visto pelo lado esquerdo; plano 4 - vista lateral direita - mostra o sólido visto pelo lado esquerdo; plano 5 - vista inferior - mostra o sólido sendo visto pelo lado de baixo; e plano 6 - vista posterior - mostra o sólido sendo visto por trás.

\subsection{Informática Básica}

Para superar a dificuldade de visualização espacial, tem-se na disciplina de Informática Básica, ministrada no primeiro módulo, um auxílio no desenvolvimento da visualização espacial, pois é 
possível, através do software Solidworks ${ }^{\circledR}$, gerar o desenho tridimensional de um sólido e a, partir dele, obter-se as vistas ortográficas com os detalhes visíveis e os detalhes ocultos das peças visualizadas.

Dessa forma, o aluno começa a compreender como todos os detalhes são representados e a comparálos com o que é aprendido na disciplina de Desenho Técnico I, começando a desenvolver a sua visualização espacial, ver Figura 2.

Figura 2 - Representação das vistas de um sólido

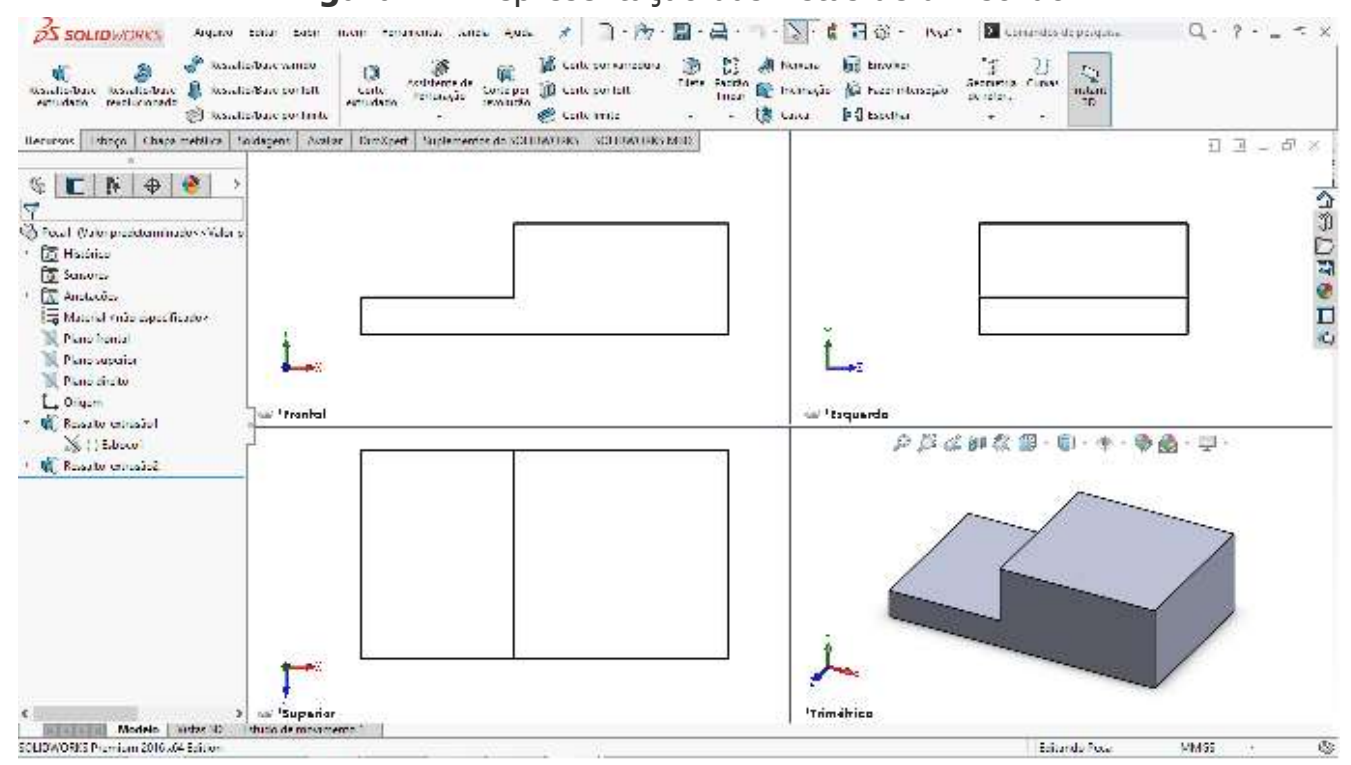

Fonte: Autor utilizando Solidworks ${ }^{\circledR}$ (2018)

\section{METODOLOGIA}

Utilizou-se a pesquisa-ação e pesquisa-participante para desenvolver este trabalho, pelo caráter de envolvimento do pesquisador e dos pesquisados no processo de intervenção e acompanhamento da proposta de trabalho conjunto entre as duas disciplinas: Desenho Técnico I e Informática Básica, o que permitiu analisar e avaliar as mudanças de aprendizagem que os estudantes viessem a apresentar como resultado deste trabalho. Na sequência segue-se com uma pesquisa de campo exploratória, utilizando como instrumento um questionário com questões abertas que foram respondidas pelos professores que trabalham com as disciplinas dentro do Curso Técnico de Mecânica.

Foi utilizada a observação-participante (VALLADARES, 2007) quando se buscou conhecer o ambiente e a metodologia utilizada para desenvolver o trabalho das duas disciplinas que se relacionam com a visualização de sólidos e sua representação gráfica para, a partir dessa observação e da análise do cenário em que se desenvolvem as aulas das disciplinas de Desenho Técnico I e Informática Básica, realizar a troca de informações com os professores envolvidos.

Com relação à Pesquisa-Ação, Nunes e Infante (1996), explicam:

A metodologia Pesquisa-Ação busca desenvolver técnicas e conhecimentos necessários ao fortalecimento das atividades valorizando o saber e a prática diária dos profissionais envolvidos, aliados aos conhecimentos teóricos e experiências adquiridas pelos pesquisadores, essa metodologia constituirá um novo saber que aponta propostas de solução dos problemas diagnosticados (NUNES e INFANTE, 1996, p. 97). 
No intuito de obter-se mais informações sobre os resultados obtidos na aprendizagem dos estudantes advindos da integração das disciplinas, realizou-se um questionário, composto de quatro perguntas abertas a serem respondidas pelos seis professores que ministram as disciplinas de Desenho Técnico I e de Informática Básica e que identificamos como professor A, B, C, D, E e F. A disciplina de Desenho Técnico I é ministrada pelos professores $A, B$ e $E$, e a disciplina de Informática Básica é ministrada pelos professores $B, C, D$, e F. Tal instrumento serviu para evidenciar as percepções dos professores envolvidos sobre o andamento do trabalho e dos ganhos obtidos no aprendizado dos estudantes.

\section{PERCEPÇÕES DOS PROFESSORES SOBRE A PROPOSTA DE TRABALHO E SUAS CONSEQUÊNCIAS}

Apresenta-se a seguir o resultado da pesquisa realizada com os professores e que constou de perguntas abertas referentes ao aprendizado que aconteceu ou era esperado ao se trabalhar em conjunto as duas disciplinas.

Ao serem perguntados sobre a proposta de utilização do software CAD, neste caso o Solidworks ${ }^{\circledR}$, ministrado na disciplina de Informática Básica, para possibilitar aos alunos de Desenho Técnico I um melhor entendimento (aprendizado) com relação à visualização espacial, obteve-se as percepções relacionadas a seguir descritas.

Corroborando com os autores Marques e Chisté (2016), o professor A acha a proposta interessante pois, segundo ele, "permite a um aluno interessado utilizar o software Solidworks ${ }^{\circledR}$ para mostrar um sólido em perspectiva e mostrar as vistas deste mesmo sólido no sistema universal de projeções e estudar as mesmas visando um possível entendimento", facilitando a visão espacial necessária para concretizar tal atividade.

O professor B acredita que "o software traz muitas ferramentas que podem auxiliar a disciplina de Desenho Técnico I, não só com a visão espacial, mas na disciplina como um todo". O professor C respondeu que este trabalho já está sendo feito, sem manifestar o resultado que está sendo proporcionado pelo mesmo.

Salientando que o desenho com os instrumentos também é base importante para o desenvolvimento do trabalho de representação gráfica, tal como afirmam Marques e Chisté (2017), o professor D é de opinião que:

O ensino do Desenho Técnico exige uma remodelação devido ao avanço da Computação Gráfica e dos programas CAD. Todavia, pelo contato com trabalhos sobre tema, é possível concluir que não se trata de uma "modernização" excluindo sua fase instrumental, visto que essa é fundamental na aprendizagem e no desenvolvimento da visão espacial, função cognitiva indispensável para a atuação de profissionais das áreas afins.

Sendo assim é necessário ir além do que mudar de instrumentos manuais para recursos tecnológicos:

É necessária uma mudança significativa nos procedimentos do professor neste novo ambiente, já que os alunos interagem mais entre si e com os colegas, estando o professor em posição de facilitador do processo. Por tudo isso, acredito que o ensino do Desenho Técnico e do CAD, integrados em um ambiente, proporcionará melhorias do ensino e, consequentemente, da aprendizagem de expressão gráfica em um curso técnico (PROFESSOR D). 
O professor E acredita ser esse o caminho a ser seguido pelas disciplinas de desenho técnico dentro do curso de mecânica, até mesmo para superar as deficiências da falta de aprendizado em geometria espacial no atual Ensino Médio, como falam Valente e Pereira (2015), Marques (2017) e Tavares (2017).

O desenho técnico "a moda antiga" não tem mais espaço na indústria atualmente. Então, o uso desde os primeiros ensinamentos de desenho técnico dos softwares CAD, não só representa uma alternativa para o melhor entendimento dos conteúdos, mas também para melhorar a qualificação de nossos técnicos (PROFESSOR E).

O conteúdo de desenho técnico é trabalhado por dois professores que usam metodologias distintas, o que, na visão do professor $F$ poderia ser unificado, ocasionando uma mudança maior e mais produtiva na forma de desenvolver o trabalho do referido conteúdo. Este afirma: "Se o professor de desenho ministrasse a aula também usando a ferramenta CAD, facilitaria para o professor e para o aluno".

Na sequência, ao serem perguntados sobre a realidade efetiva de trabalho em conjunto das duas disciplinas, tem-se as considerações abaixo:

O professor A diz apenas que sim, sem justificar ou ampliar suas visões a respeito do desenvolvimento do trabalho. Já o Professor B discorre sobre a forma de desenvolvimento do trabalho:

Bem como tem sido justificado de forma mais convincente o motivo de ministrar aulas e cobrar exercícios de Desenho Técnico (desenho feito manualmente com instrumentos e em esboço), pelo fato de justificar ao aluno que o Programa de CAD desenha aquilo que o usuário solicita (manda), o software não avalia erros de desenho e tão pouco o cumprimento das normas vigentes na área de desenho. Assuntos esses abordados na disciplina de Desenho Técnico I (PROFESSOR B).

O professor B acredita que o software traz muitas ferramentas que podem auxiliar a disciplina de Desenho Técnico I, não só com a visão espacial, mas na disciplina como um todo. Entretanto, salienta que "as duas disciplinas andam em ritmos diferentes e isso causa algumas dificuldades quando se tenta aproximar as duas".

Para o professor C, esse trabalho em conjunto é um problema, pois demanda mais tempo para o planejamento e o efetivo trabalho nas disciplinas, o que demandaria um esforço extra de professores e estudantes.

As disciplinas de Desenho Técnico I e II têm índices de reprovação muito elevados. Usar o Solidworks ${ }^{\circledR}$ em conjunto com essas disciplinas inviabilizaria o atendimento dos alunos pois não teríamos recursos docentes e físicos. Hoje, até para fazer os trabalhos de CGA em horário extraclasse os alunos têm dificuldades para encontrar o laboratório de CNC vago (PROFESSOR C).

O professor $D$ não tem aplicado o trabalho em conjunto e o professor $E$ tem trabalhado parcialmente em conjunto e é de opinião que ainda existem dificuldades e resistências a serem vencidas. $O$ professor F, quando ministra a disciplina de Informática Básica, realiza esse trabalho em conjunto.

Na terceira pergunta, sobre a constatação, por parte dos professores, sobre suas percepções a respeito da melhoria no processo de aprendizado de desenho com relação à visualização espacial por parte dos estudantes, quando adotada esta metodologia de trabalho em conjunto, o professor $\mathrm{A}$ diz que sim e ressalta que: 
A parte de desenho em CAD é uma atualidade no mercado de trabalho e muito atrativa para os alunos, dessa maneira é possível estimular os alunos a desenharem em perspectiva um rascunho via Software para então fazerem o desenho com instrumentos e em formato para ser entregue (PROFESSOR A).

O professor B, que está ministrando a disciplina de Informática Básica, como até o momento não tinha trabalhado a geração de sólidos, somente geração de esboços em 2D, não pôde ver ganhos com a aproximação entre as disciplinas. O professor C é de opinião de que não se aplica.

O professor $D$ relata que os alunos que se utilizam desse recurso têm melhor desempenho, mas como está em fase experimental, nem todos os estudantes se auxiliam do software para construírem as vistas dos desenhos.

Como ainda não temos o uso por $100 \%$ dos alunos, ficando mais a disposição dos alunos e usa aquele quem tem interesse, não conseguimos medir essa melhoria no aprendizado de forma eficiente. Mas em geral, aqueles alunos que tem a iniciativa de empregar esses recursos apresentam melhor desempenho (PROFESSOR D).

O professor F está ministrando no segundo módulo a disciplina de Computação Gráfica Aplicada I, que é uma continuidade do trabalho de Informática Básica; para esse professor, os alunos da disciplina têm muita dificuldade no desenvolvimento das tarefas propostas.

Quando questionados sobre o que poderia proporcionar um melhor aprendizado de desenho, no que se refere ao entendimento sobre visualização espacial, os professores dividem as opiniões entre os recursos didáticos utilizados e o interesse dos estudantes na utilização dos mesmos.

O professor A é de opinião de "procurar aumentar o número de exemplos e exercícios fornecidos aos alunos. Fornecer peças reais bem como as vistas destes elementos e solicitar aos alunos a interpretação destas vistas e o desenho em software da mesma".

O professor $\mathrm{B}$ acredita que trazer o sólido equivalente ao desenho em vistas para as fases iniciais melhora a visão espacial do mesmo. "No momento que o nível de complexidade das peças aumenta, o desenho 3D realizado no software tem muito a acrescentar no aprendizado do aluno" acrescenta 0 Professor B.

O professor $\mathrm{C}$ acha que devem ser aplicados exercícios, muitos exercícios, para que a prática de diferentes sólidos e peças auxiliem no desenvolvimento das habilidades e aprendizagem dos estudantes.

Já para o professor D é de suma importância procurar novas formas de estabelecer o processo de ensino-aprendizagem, buscando estimular fontes e recursos que auxiliem os estudantes e explica que isso deve ser ofertado

[ ]com a intenção de não somente melhorar a qualidade do ensino, mas também de adotar estratégias que levem o aluno a questionar e traçar novos caminhos, como forma de superar suas dificuldades cotidianas. Tratando-se dos recursos tecnológicos que nos circundam, devemos utilizá-los a nosso favor (PROFESSOR D).

O professor E considera que, em primeiro lugar, deve haver uma conscientização por parte dos estudantes sobre a importância desse conteúdo para sua vida profissional técnica. E acrescenta: 
A partir daí um comprometimento maior dos alunos, e só então assim, as ferramentas de CAD e outros recursos poderão ser empregadas com eficácia. Na minha opinião, não adianta termos acesso ao que existe de moderno em ferramentas se não tivermos alunos realmente comprometidos com o estudo. Vejo nosso aluno chegando aos cursos técnicos sem o hábito de estudar, de ler e de escrever e sem a consciência da oportunidade que tem (PROFESSOR E).

O professor $\mathrm{F}$ acha que o aluno pode entender melhor com a interação, visto que o software de CAD permite girar a peça em todas as posições, possibilitando verificar onde estão localizadas as arestas invisíveis nas vistas, e com isso já aprende a desenhar em 3D.

\section{CONSIDERAÇÕES FINAIS}

Através deste trabalho, verifica-se que a integração entre as disciplinas de Desenho Técnico I e Informática Básica possibilita a oportunidade de oferecer aos alunos recursos para o aprimoramento da visualização espacial.

Constata-se que os professores envolvidos nas duas disciplinas, apesar das especificidades de cada uma, já estão procurando essa integração.

Na percepção dos professores envolvidos, esse trabalho deve ser intensificado tanto na quantidade de desenhos a serem trabalhados quanto na extensão da metodologia para as demais disciplinas dos módulos seguintes, que usam conteúdos semelhantes com graus de dificuldade maiores.

Identifica-se ainda que o comprometimento do estudante e a sua dedicação ao cumprimento das tarefas propostas estão diretamente ligados ao seu aprendizado.

Percebe-se que o trabalho de incentivar os alunos, desde as primeiras aulas, a utilizar os recursos do Solidworks ${ }^{\circledR}$ para construir graficamente os sólidos trabalhados na disciplina de Desenho Técnico I e a utilizar esta ferramenta para visualizar um sólido e suas vistas obtidas nos planos de projeção colabora para superar as dificuldades em relação à visualização espacial.

Conclui-se de que dentro da proposta apresentada de integração das disciplinas do primeiro módulo, também está sendo procurada a ampliação do trabalho conjunto entre as disciplinas do segundo módulo, que são as de Desenho Técnico II e Computação Gráfica I, oportunizando um maior tempo de estímulo no aprendizado.

Percebe-se que outros profissionais estão preocupados com a dificuldade que os estudantes estão sentindo relacionadas à visão espacial e que se manifesta na elaboração dos desenhos técnicos, como visto nas duas pesquisas citadas durante este trabalho (Custódio, Brod e Lopes (2016) e Letícia Arruda (2018)). Sugerimos que tais pesquisas e, esta, em que se trabalhou, sejam aprofundadas para colaborar na superação das dificuldades encontradas pelos alunos neste conteúdo. 


\section{REFERÊNCIAS}

ARRUDA, L. C.. Geometria x AUTOCAD: Um Estudo de Caso no Curso de Edificações no IFSul - Câmpus Pelotas-RS. Projeto de Pesquisa. Pós-Graduação em Educação, do Instituto Federal Sulrio-grandense. Pelotas. 2018.

CUSTÓDIO, T. V., BROD, F. A. T., LOPES, J. L.. Tecnologia digital como recurso didático para potencializar o processo de aprendizagem em desenho técnico na educação profissional. Revista THEMA. Volume 13. No 2. Pág. 80 a 98. 2016.

HELLMEISTER, L. A. V., DEGANUTTI, R., ROSSI, M. A.. As Tecnologias Assistidas por Computador como Elemento de Ensino e Aprendizagem nos Cursos de Artes, Design e Engenharia. Graphica'13. Florianópolis. 2013.

IFSUL. Projeto do Curso Técnico em Mecânica Subsequente. 2008

MARQUES, J. N.. Relações entre proporção, arquitetura e matemática: abordagem em desenho técnico. Graphica 2017.

MARQUES, J. C., CHISTÉ, P. S.. O Ensino do Desenho Técnico: uma Proposta Interdisciplinar. $5^{\circ}$ Congresso Ibero-Americano em Investigação Qualitativa. Porto. Portugal. 2016.

NUNES, J. M., INFANTE, M.. Pesquisa-ação: uma metodologia de consultoria. SCIELO Books. Editora Fiocruz. 1996.

SISTEMAS de Projeções Ortogonais: <http://www.cpdee.ufmg.br/ gbarbosa/Aulas_Autocad/Desenho_T\%E9cnico/capitulo3.pdf >2008. Acesso em 17 Março. 2018.

SULZ, A. R. e SILVA, R. P.. O valor do desenho: entre a escola e a economia global. Graphica'13. Florianópolis.2013.

TAVARES, J.R.R.. A utilização dos recursos tecnológicos na disciplina de desenho: métodos de ensino contextualizados para o ensino médio. Graphica 2017.2017.

VALENTE, V. C. P. N., PEREIRA, T. T.. Aprimoramento da capacidade de visualização espacial com a utilização de hologramas. IX International Conference on Engineering and Computer Education. Zilina. Slovakia. 2015.

VALLADARES, L.. Os dez mandamentos da observação participante. Rev. bras. Ci. Soc., São Paulo, v. 22, n. 63, Feb. 2007. Disponível em <http://www.scielo.br/scielo.php?script=sci_arttext\&pid=S0102$69092007000100012 \&$ lng=en\&nrm=iso >. Acessado em 21 out. 2012.

Submissão: 25/06/2018 